\title{
DIALLEL ANALYSIS OF ELITE TOMATO LINES COMPRISING LEAF CURL VIRUS RESISTANCE GENES
}

\author{
ViJeth, S. ${ }^{1}$ - DHALIWAL, M. S. ${ }^{1}-$ JindAL, S. K. ${ }^{1}-$ GARG, N. ${ }^{2}$-KAUShIK, P. ${ }^{3 *}-$ ShaRMA, A. ${ }^{1}$ \\ ${ }^{1}$ Department of Vegetable Science, Punjab Agricultural University, Ludhiana 141 004, India \\ ${ }^{2}$ Regional Research Station, Punjab Agricultural University, Bathinda 151 001, India \\ ${ }^{3}$ Instituto de Conservación y Mejora de la Agrodiversidad Valenciana, Universitat Politècnica \\ de València, Valencia 46022, Spain \\ *Corresponding author \\ e-mail:prakau@doctor.upv.es,prashantumri@gmail.com; phone: +34-96-387-7000
}

(Received $12^{\text {th }}$ Jan 2019 ; accepted 21 Mar 2019)

\begin{abstract}
Tomato leaf curl virus is a serious threat to an autumn-winter crop of tomato in North India, causing yield losses up to $90-100 \%$. Therefore, nine resistant and one susceptible line (Punjab Chhuhara) of tomato were crossed in half-diallel fashion to develop $45 \mathrm{~F}_{1}$ hybrids. Parents and their hybrids were evaluated for yield and quality traits under natural epiphytotic field conditions. There were extensive differences in the performance of parental lines carrying similar and different resistance genes $(T y-X)$. Of three parental lines carrying $T y-2$ gene, CLN 17 performed better than 2123D and 2123C in respect of mean and general combining ability (GCA) for total yield and fruit weight. Likewise, out of three parental lines incorporating two resistance genes $(T y-1$ and $T y-2)$, the line CLN 138B had better mean values and GCA for total yield and fruit weight than CLN 138A and CLN 104. However, the parental line CLN 154 with three different resistance genes $(T y-1, T y-2, T y-3)$ showed the best GCA for total yield, and it was followed by genotype CLN $138 \mathrm{~B}$ comprising of two resistance genes (Ty-1 and $T y-2)$. However, the parental lines carrying $T y$ - 2 gene alone produced three useful hybrids expressing 19 to $28 \%$ heterosis over top parent for total yield. Four highly significant cross-combinations can be recommended for pedigree selection to develop superior inbred lines. The top parent CLN 154 yielding significantly higher than released varieties along with seven $\mathrm{F}_{1}$ hybrids manifesting 16 to $31 \%$ top parent heterosis for total yield can be recommended after multi-location evaluations. Overall, this study provides useful information on the successful leaf curl virus resistant gene combinations under the north-Indian conditions.
\end{abstract}

Keywords: begomovirus, combining ability, diallel cross, heterosis, leaf curl virus resistance, Solanum lycopersicum

\section{Introduction}

Tomato (Solanum lycopersicum L.) is one of the most important vegetable crops of India and the world. India contributes $11.1 \%$ to world tomato production and is the second largest tomato producer in the world after China (FAOSTAT, 2018). In North Indian plains, the autumn-winter crop of tomato is transplanted during second fortnight of August. The main constraint in this crop is the incidence of tomato leaf curl virus (ToLCV) which may cause yield losses up to 90-100\% (Gaikwad et al., 2011a; Kaushik and Dhaliwal, 2018; Moriones et al., 2017). The disease is transmitted by whitefly (Bemisia tabaci Gennadius), the population of which is usually high during July to October under Punjab conditions (Kaushik and Dhaliwal, 2018). Management of the whitefly using systemic insecticides has proved futile because of rapid turnover rate of the vector population in the tomato crop (Shankarappa et al., 2008; Singh et al., 2015) and frequent development of resistance against insecticides (Horowitz et al., 2005). Therefore, breeding for disease resistance is the best approach to control this disease 
(Picó et al., 1996). In this direction, the Punjab Agricultural University has so far developed and released three open-pollinated resistant varieties of tomato viz., Punjab Varkha Bahar 1 (PVB-1), Punjab Varkha Bahar 2 (PVB-2) (Cheema and Dhaliwal, 2010) and Punjab Varkha Bahar 4 (PVB-4). However, it is important to develop $F_{1}$ hybrids resistant to ToLCV because tomato hybrids generally outperform openpollinated varieties and are therefore widely cultivated by farmers in India (Prasanna et al., 2015). Although a few hybrid combinations have been identified by Shankarappa et al. (2008) for cultivation in South India, there is a need to develop and identify resistant hybrids for each geographical region because of the occurrence of a different isolate of the virus in each area. The earlier work on the geographical distribution of tomato leaf curl begomovirus species within India suggested that viruses with bipartite genomes (DNA-A and DNA-B components) were prevalent in North India while those with monopartite genomes (DNA-A) were found in South India (Hong and Harrison, 1995; Kirthi et al., 2002; Muniyappa et al., 2000).

Tomato leaf curl virus is a geminivirus belonging to the Begomovirus genus of the family Geminiviridae (Varma and Malathi, 2003). Five different leaf curl viruses predominantly infecting tomato crop have been reported in India. These include tomato leaf curl New Delhi virus (ToLCNDV), tomato leaf curl Palampur virus (ToLCPMV), tomato leaf curl Bangalore virus (ToLCBV), tomato leaf curl Karnataka virus (ToLCKV) and tomato leaf curl Gujarat virus (ToLCGV). A recent survey and molecular characterization of ToLCV revealed that three begomovirus species, viz., ToLCNDV, ToLCPMV and ToLCKV are predominant under Punjab conditions (Gaikwad et al., 2011b; Kaushik et al., 2015).

Six $T y$-genes ( $T y-1$ to $T y-6)$ conferring the variable degree of resistance to ToLCV are available in cultivated tomato (Hanson et al., 2016). Two partially dominant genes Ty-1 and Ty-3 are mapped on chromosome 6 (Ji et al., 2007a; Zamir et al., 1994) and are reported to be at the same locus (Verlaan et al., 2013). A dominant gene $T y-2$ was mapped on chromosome 11 (Hanson et al., 2006). The Ty-4 is a minor QTL on chromosome 3 that accounted for only 16\% variation in symptom severity ( $\mathrm{Ji}$ et al., 2009). Besides, a recessive gene ty-5 was mapped on chromosome 4 (Hutton et al., 2012). Of late, Hutton and Scott (2014) have identified a Ty-6 gene on chromosome 10 that confers resistance against the bipartite TYLCV. Of these genes, the dominant $T y-2$ and partially dominant $T y-3$ carrying lines could be potential resources for developing resistant or tolerant hybrids due to the nature of their gene action (Prasanna et al., 2015). The present study was, therefore, undertaken to evaluate ten parental lines along with their $45 \mathrm{~F}_{1}$ hybrids incorporating $T y-1, T y-2$ and $T y-3$ genes in respect of yield and quality traits under natural field conditions (against local isolate of ToLCV) and to identify superior inbred lines and $\mathrm{F}_{1}$ hybrids based on their combining ability, heterosis and per se performance for yield and other horticultural traits.

\section{Materials and methods}

The present investigations were conducted at Vegetable Research Farm, Punjab Agricultural University, Ludhiana, coordinates at $30^{\circ} 55^{\prime}$ North latitude, $75^{\circ} 54^{\prime}$ 'East longitude and $247 \mathrm{~m}$ altitude. The plant material comprised ten advance generation lines of tomato (Table 1). Of these, nine lines, viz., PVB-2, CLN 138A, CLN 138B, CLN 104, CLN 17, 2123D, 2123C, PVB-1 and CLN 154, had a varying degree of resistance to ToLCV. The tenth parental line, viz., Punjab Chhuhara, was susceptible to ToLCV 
(Gaikwad et al., 2011a; Kaushik and Dhaliwal, 2018). All the ten parental lines were crossed manually during February-March, 2014 in a diallel mating design (Griffing, 1956) without reciprocals to generate $45 \mathrm{~F}_{1}$ hybrids. The seed of all the 55 entries were

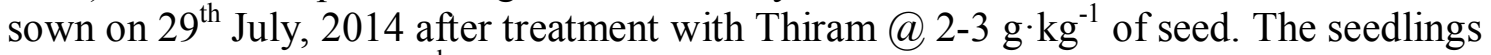
were transplanted on $2^{\text {nd }}$ September, 2014 in a randomized complete block design (RCBD) with three replications. Ten plants of each entry were planted in a single row. Planting was done on one side of the raised beds, and row $\times$ plant spacing was maintained at $1.35 \mathrm{~m} \times 0.30 \mathrm{~m}$. The beds were covered with steel gray plastic mulch of $25 \mu \mathrm{m}$ thickness. The insecticides were not sprayed in the trial so as to enhance the population of whitefly. To increase inoculum pressure, one row of ToLCV susceptible cv. Punjab Chhuhara was planted after every 10 rows in the experimental plots.

Table 1. Particulars of ten parental lines of tomato evaluated in half-diallel for yield and quality traits

\begin{tabular}{c|c|c|c|c}
\hline Parental line & Pedigree/alternate name & Resistance gene(s) & Source & References \\
\hline PVB-2 & Punjab Varkha Bahar-2 & Unknown & PAU & - \\
\hline CLN 138A & CLN 3022 F 2 -138-6-2-0 & Ty-1, Ty-2 & AVRDC & Zamir et al. (1994) \\
\hline CLN 138B & CLN 3022 F $2-138-6-7-0$ & Ty-1, Ty-2 & AVRDC & $\begin{array}{c}\text { Hanson et al. (2006); } \\
\text { Zamir et al. (1994) }\end{array}$ \\
\hline CLN 104 & CLN 3024 F $-104-48-1-0$ & Ty-1, Ty-2 & AVRDC & $\begin{array}{c}\text { Hanson et al. (2006); } \\
\text { Zamir et al. (1994) }\end{array}$ \\
\hline CLN 17 & CLN 3008 F $2-17-10-0$ & Ty-2 & AVRDC & Hanson et al. (2006) \\
\hline 2123D & CLN 2123D & Ty-2 & AVRDC & Hanson et al. (2006) \\
\hline 2123C & CLN 2123C & Ty-2 & AVRDC & Hanson et al. (2006) \\
\hline PVB-1 & Punjab Varkha Bahar-1 & Unknown & PAU & - \\
\hline CLN 154 & CLN 3022 F $2-154-11-11-0$ & Ty-1, Ty-2, Ty-3 & AVRDC & $\begin{array}{c}\text { Hi et al. (2007b); } \\
\text { Zamir et al. (1994) }\end{array}$ \\
\hline PC & Punjab Chhuhara & Nil & PAU & -
\end{tabular}

PAU: Punjab Agricultural University, Ludhiana, India; AVRDC: The World Vegetable Center, Shanhua, Tainan, Taiwan

The plants were characterized based on the scale shown in Figure 1. After that, potential disease incidence (PDI) was determined with the formula as (number of infected plants/total number of plants) $\times 100$ (Kaushik and Dhaliwal, 2018). The observations were recorded for ten traits. Total yield $\left(\mathrm{kg} \mathrm{plant}^{-1}\right)$ was calculated by adding the weight of fruits obtained from all pickings and dividing by number of plants per entry per replication. A random sample of five fruits from second picking was taken and fruit weight $(\mathrm{g})$ was estimated by dividing the weight of the sample with the number of fruits in the sample. Five randomly selected fruits from second harvest were used to determine polar diameter of fruit (distance between stem end and blossom end of the fruit), equatorial diameter of fruit (transversal distance of the fruit) and pericarp thickness (after dissecting the equatorial plane of the fruit) with the help of an Absolute Digimatic Vernier Caliper (Mitutoyo Corporation) and average was worked out. The same fruits were used to count the number of locules per fruit and average was worked out. For quality evaluation, five red ripe fruits were taken from second picking and cut longitudinally. Juice was squeezed from the blossom end and filtered through a double 
layer of muslin cloth. Total soluble solids ( ${ }^{\circ}$ Brix), titratable acidity (mg/100 ml juice), lycopene (mg/100 g fresh weight) and dry matter (\%) were estimated according to the procedure described elsewhere (Garg and Cheema, 2011).

Analysis of variance was performed for each trait, and the differences among mean values of all 55 entries for each trait were tested for significance using standard error of difference. Diallel analysis was carried out according to Griffing (1956) method II, model 1 (fixed effect model) to estimate general combining ability (GCA) and specific combining ability (SCA) effects. Heterosis over mid-parent (MPH), better parent (BPH) and top parent (TPH) was estimated and tested for significance using standard formulas (Garg and Cheema, 2011).

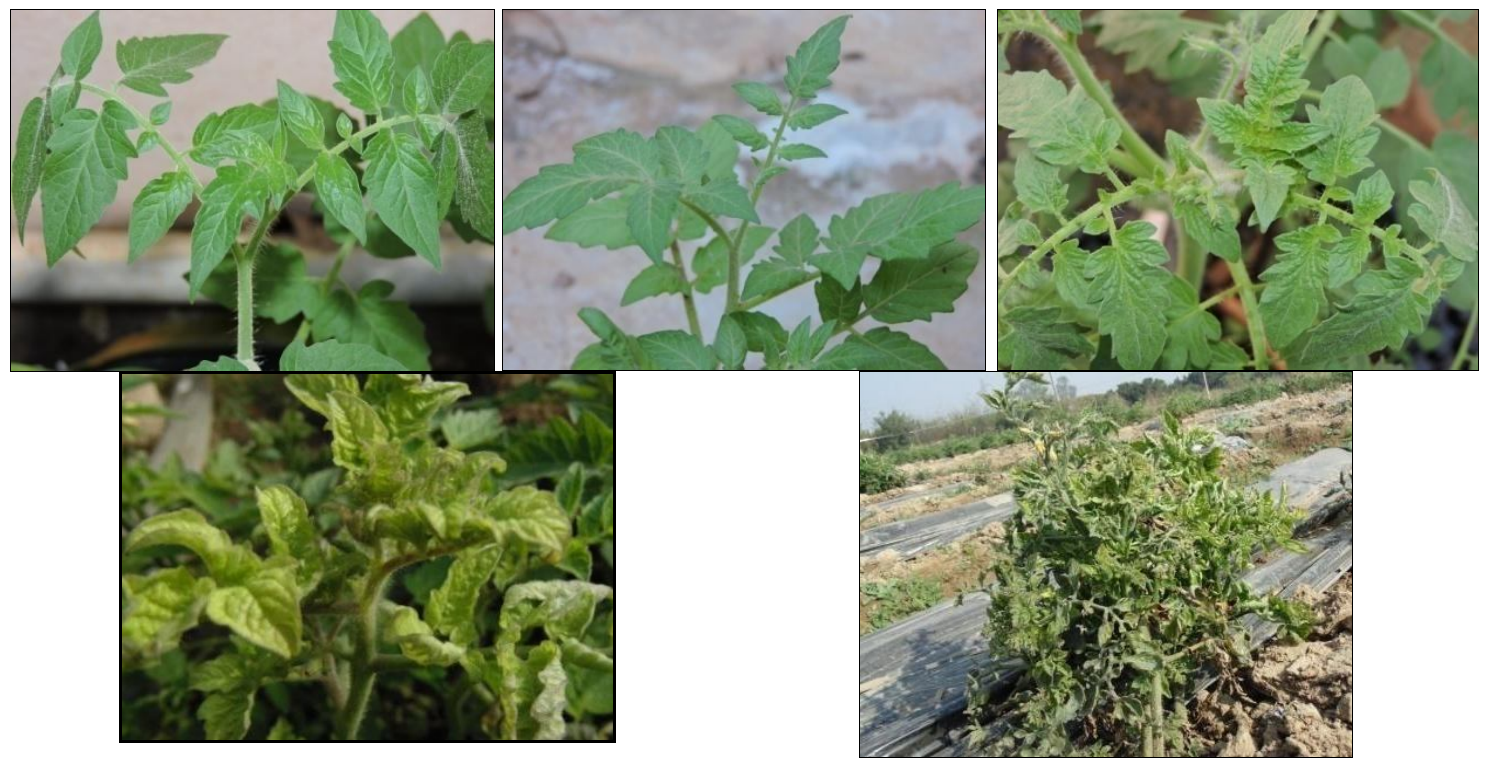

Figure 1. ToLCV symptoms severity rating in tomato on 0-4 scale, where $0=$ Symptomless, $1=$ Resistant, $2=$ Tolerant, $3=$ Susceptible and $4=$ Highly susceptible

\section{Results}

Under open field conditions, seven out of ten parents showed a nil PDI (\%) (Fig. 2). Remaining, one parent PC was susceptible to ToLCV infestation and remaining two parents showed the PDI (\%) of 35-45\% (Fig. 2). Whereas, twenty hybrids out of total forty-five demonstrated nil infection (Fig. 2). While for the remaining hybrids the PDI (\%) ranged from $25-80 \%$ (Fig. 2). The analysis of variance for the experimental design revealed that the mean squares for genotypes were significant for all the traits indicating potential genetic differences among genotypes i. e. parents and their $\mathrm{F}_{1}$ hybrids. The analysis of variance for combining ability revealed that variances for GCA and SCA were significant for all the traits indicating the importance of additive as well as nonadditive types of gene effects in the inheritance of the traits studied. The GCA effects represent the additive nature of gene action. A high general combiner parent is characterized by its better breeding value when crossed with several other parents. Besides, mean performance of the parent is also considered in unison with GCA since the former offers reliability to GCA as a guide to the selection of parent (Sharma, 2006; Singh et al., 2014). Four parental lines viz., CLN 154, CLN 138B, PVB-2 and PVB-1 had shown significant positive values of GCA for total yield along with correspondingly 
high mean yield (Table 2) reflecting their true breeding value. These lines also showed significant and positive GCA for fruit weight and quality traits. The GCA of parental lines was in consonance with their mean performance. For example, parental lines having high GCA for total yield had high mean yield too. However, there were differences in the combining ability of parental lines carrying the same resistance gene. For example, CLN 17, 2123D and 2123C had the same resistance gene Ty-2, but CLN 17 was a better combiner for total yield than the other two parental lines. Likewise, the line CLN 138B was a better combiner than CLN 138A and CLN 104 although all these three lines incorporated same resistant genes, viz., $T y-1$ and $T y-2$.

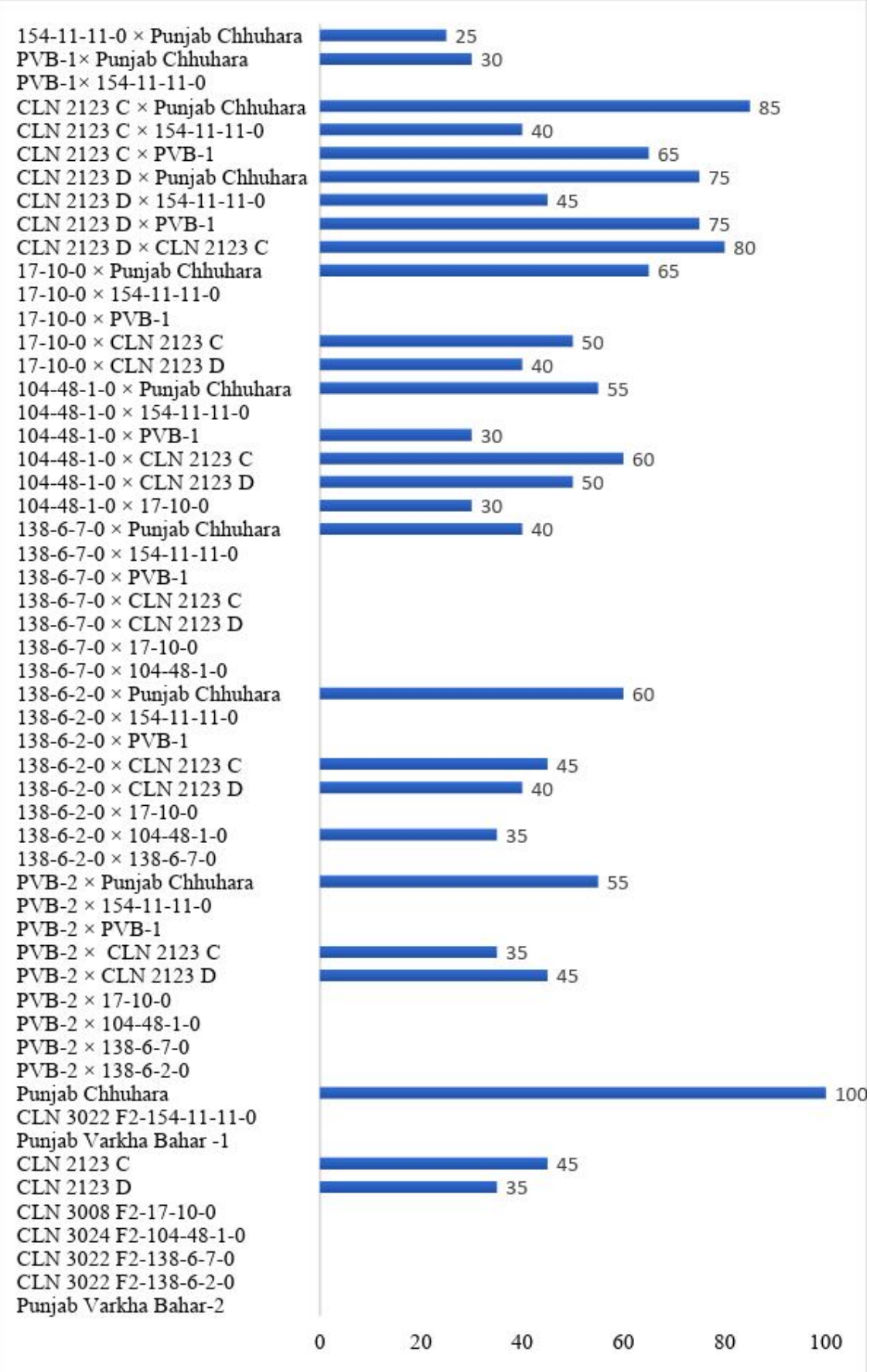

Figure 2. Per cent disease incidence (PDI) reaction of parents and their hybrids 
Table 2. General combining ability (GCA) effects and mean values of ten parental lines of tomato evaluated in half-diallel for yield and quality traits

\begin{tabular}{|c|c|c|c|c|c|c|c|c|c|c|}
\hline \multirow{2}{*}{$\begin{array}{l}\text { Parental } \\
\text { line }\end{array}$} & \multicolumn{2}{|c|}{$\begin{array}{l}\text { Total yield } \\
\left(\text { kg plant }^{-1}\right)\end{array}$} & \multicolumn{2}{|c|}{ Fruit weight (g) } & \multicolumn{2}{|c|}{$\begin{array}{c}\text { Number of } \\
\text { locules }\end{array}$} & \multicolumn{2}{|c|}{$\begin{array}{c}\text { Pericarp } \\
\text { thickness }(\mathrm{mm})\end{array}$} & \multicolumn{2}{|c|}{$\begin{array}{c}\text { Polar diameter } \\
(\mathbf{c m})\end{array}$} \\
\hline & GCA & Mean & GCA & Mean & GCA & Mean & GCA & Mean & GCA & Mean \\
\hline PVB-2 & $0.22 *$ & 2.13 & $6.15^{*}$ & 96.67 & $0.52 *$ & 4.22 & -0.10 & 5.58 & $-0.09 *$ & 5.61 \\
\hline CLN 138A & -0.03 & 1.17 & $-3.41 *$ & 67.67 & $-0.28 *$ & 3.03 & $-0.65^{*}$ & 4.33 & -0.05 & 4.88 \\
\hline CLN 138B & $0.42^{*}$ & 1.97 & $12.27 *$ & 102.00 & $0.26^{*}$ & 3.50 & $1.18^{*}$ & 6.33 & $0.49^{*}$ & 6.30 \\
\hline CLN 104 & -0.07 & 1.30 & -0.07 & 61.33 & -0.03 & 2.92 & $-0.42 *$ & 3.57 & $-0.29 *$ & 3.94 \\
\hline CLN 17 & 0.07 & 1.60 & $1.36^{*}$ & 86.33 & $0.25 *$ & 4.00 & $0.43 *$ & 6.57 & -0.03 & 5.93 \\
\hline $2123 \mathrm{D}$ & $-0.20 *$ & 1.20 & $-4.32 *$ & 64.67 & $-0.52 *$ & 2.93 & $-0.71 *$ & 3.77 & $-0.18 *$ & 4.67 \\
\hline $2123 \mathrm{C}$ & $-0.34 *$ & 0.97 & $-8.74 *$ & 60.33 & $-0.53 *$ & 2.80 & $-0.43^{*}$ & 4.10 & $-0.12 *$ & 4.44 \\
\hline PVB-1 & $0.14^{*}$ & 1.97 & $7.71^{*}$ & 94.00 & $0.45^{*}$ & 3.98 & $0.42 *$ & 5.60 & $0.14^{*}$ & 6.00 \\
\hline CLN 154 & $0.43 *$ & 2.50 & $3.54 *$ & 89.33 & $0.53 *$ & 4.00 & $0.23 *$ & 6.20 & $0.09 *$ & 5.50 \\
\hline $\mathrm{PC}$ & $-0.63 *$ & 0.26 & $-14.50^{*}$ & 34.33 & $-0.65^{*}$ & 2.30 & 0.05 & 4.60 & 0.02 & 4.92 \\
\hline $\begin{array}{c}* * \mathrm{CD}\left(\mathrm{g}_{\mathrm{i}}\right) \\
(\mathrm{P} \leq 0.05)\end{array}$ & 0.07 & & 1.11 & & 0.08 & & 0.13 & & 0.09 & \\
\hline $\begin{array}{c}\mathrm{CD}\left(\mathrm{g}_{\mathrm{i}}-\mathrm{g}_{\mathrm{j}}\right) \\
(\mathrm{P} \leq 0.05)\end{array}$ & 0.10 & & 1.65 & & 0.12 & & 0.19 & & 0.14 & \\
\hline $\begin{array}{c}\mathrm{CD} \\
(\mathrm{P} \leq 0.05)\end{array}$ & & 0.36 & & 5.69 & & 0.43 & & 0.65 & & 0.50 \\
\hline
\end{tabular}

*Significant at $\mathrm{P} \leq 0.05, * *$ Critical difference $(\mathrm{CD})$

Table 2. contd.

\begin{tabular}{|c|c|c|c|c|c|c|c|c|c|c|}
\hline \multirow[t]{2}{*}{$\begin{array}{l}\text { Parental } \\
\text { line }\end{array}$} & \multicolumn{2}{|c|}{$\begin{array}{c}\text { Equatorial } \\
\text { diameter }(\mathrm{cm})\end{array}$} & \multicolumn{2}{|c|}{$\begin{array}{l}\text { Total soluble } \\
\text { solids ( }{ }^{\circ} \text { Brix) }\end{array}$} & \multicolumn{2}{|c|}{$\begin{array}{c}\text { Titratable } \\
\text { acidity (mg/100 } \\
\text { ml) }\end{array}$} & \multicolumn{2}{|c|}{$\begin{array}{l}\text { Lycopene } \\
\text { (mg/100 g) }\end{array}$} & \multicolumn{2}{|c|}{ Dry matter $(\%)$} \\
\hline & GCA & Mean & GCA & Mean & GCA & Mean & GCA & Mean & GCA & Mean \\
\hline PVB-2 & $0.46^{*}$ & 5.97 & 0.01 & 4.17 & $-0.04 *$ & 0.53 & $0.53^{*}$ & 3.20 & $0.14^{*}$ & 5.01 \\
\hline CLN 138A & $-0.10^{*}$ & 4.35 & -0.02 & 3.85 & $-0.03 *$ & 0.66 & $0.27^{*}$ & 3.57 & 0.03 & 4.69 \\
\hline CLN 138B & $0.65^{*}$ & 6.09 & $0.11^{*}$ & 4.30 & $-0.08 *$ & 0.43 & $0.09^{*}$ & 3.00 & $0.15^{*}$ & 4.17 \\
\hline CLN 104 & $-0.34 *$ & 3.62 & $0.42 *$ & 4.80 & $0.10^{*}$ & 0.75 & $0.56^{*}$ & 3.97 & $0.22 *$ & 3.04 \\
\hline CLN 17 & -0.06 & 5.59 & -0.01 & 3.71 & $0.07^{*}$ & 0.66 & -0.06 & 3.30 & 0.07 & 4.44 \\
\hline $2123 \mathrm{D}$ & $-0.50 *$ & 4.19 & $-0.13 *$ & 2.97 & $-0.06^{*}$ & 0.69 & $-1.14^{*}$ & 2.13 & $-0.48^{*}$ & 3.93 \\
\hline $2123 \mathrm{C}$ & $-0.28 *$ & 4.18 & $-0.38^{*}$ & 3.30 & $0.04 *$ & 0.65 & $-0.90^{*}$ & 1.96 & $-0.59^{*}$ & 3.28 \\
\hline PVB-1 & $0.31^{*}$ & 6.24 & $-0.17 *$ & 4.50 & $-0.01 *$ & 0.48 & $0.40^{*}$ & 3.50 & $0.16^{*}$ & 4.90 \\
\hline CLN 154 & $0.81^{*}$ & 5.45 & $0.40^{*}$ & 4.47 & $-0.06^{*}$ & 0.47 & $0.24^{*}$ & 3.87 & $-0.16^{*}$ & 4.32 \\
\hline PC & $-0.32 *$ & 4.22 & $-0.23^{*}$ & 4.11 & $0.08^{*}$ & 0.51 & 0.00 & 3.10 & $0.48^{*}$ & 4.40 \\
\hline $\begin{array}{c}* * \mathrm{CD}\left(\mathrm{g}_{\mathrm{i}}\right) \\
(\mathrm{P} \leq 0.05)\end{array}$ & 0.08 & & 0.10 & & 0.01 & & 0.08 & & 0.11 & \\
\hline $\begin{array}{c}\mathrm{CD}\left(\mathrm{g}_{\mathrm{i}}-\mathrm{g}_{\mathrm{j}}\right) \\
(\mathrm{P} \leq 0.05)\end{array}$ & 0.12 & & 0.14 & & 0.02 & & 0.12 & & 0.16 & \\
\hline $\begin{array}{c}\mathrm{CD} \\
(\mathrm{P} \leq 0.05)\end{array}$ & & 0.43 & & 0.50 & & 0.07 & & 0.43 & & 0.56 \\
\hline
\end{tabular}

*Significant at $\mathrm{P} \leq 0.05, * *$ Critical difference $(\mathrm{CD})$ 
The $\mathrm{F}_{1}$ hybrids had a higher mean and wider range than their parental lines for all the traits. However, the maximum increase was recorded in total yield $(47.68 \%)$ followed by fruit weight $(20.27 \%)$, number of locules $(15.13 \%)$ and titratable acidity $(13.73 \%)$. For other traits, the average heterosis was less than 10\% (Table 3). Heterosis of both positive and negative magnitudes was observed for all the traits. For total yield, 38, 19 and 7 hybrids exhibited significant positive values of MPH, BPH and TPH, respectively. Not even a single hybrid manifested significant negative MPH whereas only 3 hybrids recorded significant negative BPH (Table 4). The promising hybrids along with their TPH were CLN 138A $\times$ CLN 138B (31.20\%), CLN 138B $\times 2123 \mathrm{C}$ (28.00\%), CLN 138A $\times$ CLN 154 (24.00\%), CLN 138B × PVB-1 (22.80\%), CLN $17 \times$ PVB-1 (20.80\%), CLN 138B × 2123D (18.80\%) and CLN $104 \times$ CLN $154(16.00 \%)$. All of these seven hybrids had significant positive SCA effects (Table 4) reflecting the importance of non-additive gene action in heterosis manifestation for total yield. For fruit weight, 34, 16 and 5 hybrids exhibited significant positive values of MPH, BPH and TPH, respectively. Whereas, there were 3, 15 and 27 hybrids manifesting significant negative values of MPH, BPH and TPH, respectively (Table 4). Out of five hybrids exceeding the top parent for fruit weight, three (CLN 138B $\times$ PVB-1, CLN $138 \mathrm{~A} \times \mathrm{CLN} 154$ and CLN $138 \mathrm{~B} \times 2123 \mathrm{C}$ ) were the same that recorded significant positive TPH for total yield revealing that increase in total yield was accompanied by an increase in fruit weight. All of these five hybrids manifested significant positive SCA values (Table 4) signifying the role of non-additive gene action in the expression of heterosis for fruit weight.

Table 3. Mean, and range of parental lines and their $F_{1}$ hybrids along with average heterosis for yield and quality traits in ten parental lines of tomato evaluated in half-diallel

\begin{tabular}{|c|c|c|c|c|c|c|c|c|c|c|}
\hline Parameter & $\begin{array}{l}\text { Total yield } \\
\left.\text { (kg plant }^{-1}\right)\end{array}$ & $\begin{array}{c}\text { Fruit } \\
\text { weight } \\
\text { (g) }\end{array}$ & $\begin{array}{l}\text { Number } \\
\text { of locules }\end{array}$ & $\begin{array}{c}\text { Pericarp } \\
\text { thickness } \\
(\mathbf{m m})\end{array}$ & $\begin{array}{c}\text { Polar } \\
\text { diameter } \\
(\mathrm{cm})\end{array}$ & $\begin{array}{c}\text { Equatorial } \\
\text { diameter } \\
(\mathrm{cm})\end{array}$ & $\begin{array}{c}\text { TSS } \\
\left({ }^{\circ} \text { Brix }\right)\end{array}$ & $\begin{array}{c}\text { Titratable } \\
\text { acidity } \\
(\mathrm{mg} / 100 \mathrm{ml})\end{array}$ & $\begin{array}{l}\text { Lycopene } \\
(\mathrm{mg} / 100 \mathrm{~g})\end{array}$ & $\begin{array}{c}\text { Dry } \\
\text { matter } \\
(\%)\end{array}$ \\
\hline Parental mean & 1.51 & 75.67 & 3.37 & 5.07 & 5.22 & 4.99 & 4.02 & 0.51 & 3.16 & 4.22 \\
\hline Parental range & $\begin{array}{l}0.26- \\
2.50\end{array}$ & $\begin{array}{l}34.33- \\
102.00\end{array}$ & $\begin{array}{l}2.30- \\
4.22\end{array}$ & $\begin{array}{l}3.57- \\
6.57\end{array}$ & $\begin{array}{c}3.94- \\
6.30\end{array}$ & $\begin{array}{l}3.62- \\
6.24\end{array}$ & $\begin{array}{c}2.97- \\
4.80\end{array}$ & $\begin{array}{l}0.43- \\
0.75\end{array}$ & $\begin{array}{l}1.96- \\
3.97\end{array}$ & $\begin{array}{c}3.04- \\
5.01\end{array}$ \\
\hline Hybrid mean & 2.23 & 91.01 & 3.88 & 5.32 & 5.27 & 5.16 & 4.30 & 0.58 & 3.40 & 4.32 \\
\hline Hybrid range & $\begin{array}{l}0.61- \\
3.28\end{array}$ & $\begin{array}{l}59.67- \\
129.00\end{array}$ & $\begin{array}{c}2.23- \\
5.70\end{array}$ & $\begin{array}{l}3.13- \\
8.17\end{array}$ & $\begin{array}{l}4.30- \\
6.50\end{array}$ & $\begin{array}{c}3.40- \\
7.20\end{array}$ & $\begin{array}{c}2.90- \\
5.67\end{array}$ & $\begin{array}{l}0.27- \\
0.82\end{array}$ & $\begin{array}{l}1.03- \\
5.50\end{array}$ & $\begin{array}{c}2.77- \\
5.80\end{array}$ \\
\hline $\begin{array}{c}\text { Average } \\
\text { heterosis }(\%)\end{array}$ & 47.68 & 20.27 & 15.13 & 4.93 & 0.96 & 3.41 & 6.97 & 13.73 & 7.59 & 2.37 \\
\hline
\end{tabular}

Of 45 hybrids, 15 involved low $\times$ low combiner parents for total yield. Although none of these exhibited significant positive TPH, there were 13 and 8 hybrids manifesting 27.94-130.77\% and 34.38-102.86\% $\mathrm{MPH}$ and $\mathrm{BPH}$, respectively. This result raises the importance of low combiner parents in heterosis breeding. On the contrary, there were a few cross-combinations viz., PVB-2 $\times$ CLN 138B, PVB-2 $\times$ CLN 154, CLN 138B $\times$ CLN 154, and PVB-1 $\times$ CLN 154, which could not express significant heterosis for commercialization but involved parents having medium $\times$ high and high $\times$ high combiner parents for total yield. In the present study, three parental lines were incorporating $T y-2$ gene viz., CLN 17, 2123D and 2123C. The yield potential of these three lines was significantly different. The line CLN 17 yielded $1.60 \mathrm{~kg} \mathrm{plant}^{-1}$ which was substantially higher than 2123D (1.20 kg plant $\left.{ }^{-1}\right)$ 
and $2123 \mathrm{C}\left(0.97 \mathrm{~kg}\right.$ plant $\left.^{-1}\right)$. Likewise, the fruit weight of CLN 17 was $86.33 \mathrm{~g}$ which was significantly higher than that of 2123D (64.67g) and $2123 \mathrm{C}(60.33 \mathrm{~g})$. The line CLN 17 performed better in hybrid combinations than 2123D and 2123C did, making it a better general combiner for fruit yield and fruit weight. Hence, the line CLN 17 performed better than 2123D and 2123C in respect of yield, fruit weight and GCA for yield \& fruit weight. However, there were three promising $\mathrm{F}_{1}$ hybrids involving these lines viz., CLN 138B × 2123C, CLN 138B × 2123D and CLN $17 \times$ PVB-1 which recorded $28.00,18.80$ and $20.80 \%$ increase in total yield over top parent, respectively. Therefore, the parental lines carrying $T y$-2 gene alone should not be considered useless and may yield useful hybrid combinations and hence may be included in tomato breeding programs aimed at developing resistance to ToLCV.

There were three parental lines viz., CLN 138A, CLN 138B and CLN 104 carrying two resistant genes $T y-1$ and $T y-2$. Of these three lines, the yield and fruit weight of CLN 138B (1.97 kg plant ${ }^{-1}$ and $\left.102.00 \mathrm{~g}\right)$ was significantly higher than that of CLN $104\left(1.30 \mathrm{~kg} \mathrm{plant}^{-1}\right.$ and $\left.61.33 \mathrm{~g}\right)$ and CLN 138A (1.17 kg plant ${ }^{-1}$ and $\left.67.67 \mathrm{~g}\right)$. The line CLN 138B had significant positive GCA for yield and fruit weight whereas the other two lines exhibited negative values of GCA for these two traits. So, the line CLN 138B was better than CLN 138A and CLN 104 concerning yield, fruit weight and GCA for yield \& fruit weight. The parental line CLN 154 incorporated three resistant genes $T y-1, T y-2$ and $T y-3$ whereas the lines PVB-1 and PVB-2 had unknown resistance genes. The fruit weight of these three lines was at par with each other. However, CLN 154 yielded $2.50 \mathrm{~kg}$ plant $^{-1}$ which was significantly higher than that of PVB-2 (2.13 kg plant $\left.{ }^{-1}\right)$ and PVB-1 (1.97 kg plant $\left.{ }^{-1}\right)$. Although all of these three lines had significant positive values of GCA for yield and fruit weight, CLN 154 was better combiner for yield and PVB-1 for fruit weight. The superior performance of CLN 154 as compared to released cultivars (PVB-1 and PVB-2) offers the scope of its commercial release and is, therefore, recommended for multi-location testing across the state.

High yield is one of the most important breeding objectives under disease epiphytotic conditions. Among parents, the line CLN 154 incorporating three resistance genes was the highest yielder $\left(58.65 \mathrm{t} \mathrm{ha}^{-1}\right)$. To cover up the cost of hybrid seeds, it was desirable to identify hybrids yielding significantly higher than the top parent. In this context, seven hybrids exhibiting 16.00-31.20\% heterosis over top parent for total yield were identified. These seven hybrids also manifested 16.00$66.95 \%$ heterosis over better parent and 52.63-108.92\% over mid-parent for total yield. The fruit weight of promising hybrids varied from $82.33 \mathrm{~g}$ to $117.33 \mathrm{~g}$ which is well within acceptable limits and comparable with that of top parent CLN 154 (89.33 g). The number of locules and pericarp thickness of promising hybrids ranged from 3.33-5.40 and 4.23-7.37 mm, respectively, which were comparable to top parent CLN $154(4.0,6.20 \mathrm{~mm})$. The fruit shape of all the promising hybrids was round just like the top parent and round-fruited hybrids are highly acceptable for table purpose. Similarly, the total soluble solids, titratable acidity, lycopene and dry matter of promising hybrids varied from 3.47-5.00 ${ }^{\circ}$ Brix, 0.32-0.59 mg/100 ml, 3.03-5.50 $\mathrm{mg} / 100 \mathrm{~g}$ and $3.87-5.30 \%$, respectively, which were comparable with CLN 154 $\left(4.47^{\circ}\right.$ Brix, $\left.0.47 \mathrm{mg} / 100 \mathrm{ml}, 3.87 \mathrm{mg} / 100 \mathrm{~g}, 4.32 \%\right)$. The desirable horticultural traits of the promising hybrids enhance the feasibility of their commercial acceptability. 
Table 4. Specific combining ability (SCA) and heterosis over mid-parent (MPH), better parent (BPH) and top parent (TPH) exhibited by forty five $F_{1}$ hybrids of ten parental lines of tomato crossed in half-diallel for total yield and fruit weight

\begin{tabular}{|c|c|c|c|c|c|c|c|c|c|c|}
\hline \multirow{2}{*}{$F_{1}$ hybrid } & \multicolumn{5}{|c|}{ Total yield } & \multicolumn{5}{|c|}{ Fruit weight } \\
\hline & SCA & $\begin{array}{l}\text { GCA of } \\
\text { parents }\end{array}$ & MPH & BPH & TPH & SCA & $\begin{array}{l}\text { GCA of } \\
\text { parents }\end{array}$ & МРН & BPH & TPH \\
\hline PVB-2 $\times$ CLN 138A & 0.11 & $\mathrm{M} \times \mathrm{L}$ & $45.45^{*}$ & 12.5 & -4 & $-10.29 *$ & $\mathrm{H} \times \mathrm{L}$ & -1.83 & $-16.55^{*}$ & $-20.91 *$ \\
\hline PVB-2 $\times$ CLN 138B & $-0.81 *$ & $\mathrm{M} \times \mathrm{H}$ & -5.85 & -9.38 & $-22.80 *$ & $-18.97 *$ & $\mathrm{H} \times \mathrm{H}$ & $-11.74 *$ & $-14.05 *$ & $-14.05 *$ \\
\hline PVB-2 × CLN 104 & -0.09 & $\mathrm{M} \times \mathrm{L}$ & $26.53 *$ & 1.56 & -13.2 & 0.03 & $\mathrm{H} \times \mathrm{L}$ & $19.41^{*}$ & -2.41 & $-7.52 *$ \\
\hline PVB-2 × CLN 17 & 0.11 & $\mathrm{M} \times \mathrm{L}$ & $34.05^{*}$ & $17.19 *$ & 0 & $6.26^{*}$ & $\mathrm{H} \times \mathrm{M}$ & $11.48^{*}$ & 5.52 & 0 \\
\hline PVB-2 $\times 2123 D$ & 0.08 & $\mathrm{M} \times \mathrm{L}$ & $32.13^{*}$ & 3.12 & -12 & $4.94 *$ & $\mathrm{H} \times \mathrm{L}$ & $17.76^{*}$ & -1.72 & $-6.86^{*}$ \\
\hline PVB- $2 \times 2123 \mathrm{C}$ & $0.62 *$ & $\mathrm{M} \times \mathrm{L}$ & $67.74 *$ & $21.88^{*}$ & 4 & -0.3 & $\mathrm{H} \times \mathrm{L}$ & $8.70^{*}$ & $-11.72 *$ & $-16.34 *$ \\
\hline PVB-2 × PVB-1 & 0.05 & $\mathrm{M} \times \mathrm{M}$ & $22.44 *$ & $17.66^{*}$ & 0.4 & $17.25^{*}$ & $\mathrm{H} \times \mathrm{H}$ & $25.17 *$ & $23.45^{*}$ & $16.99 *$ \\
\hline PVB-2 $\times$ CLN 154 & -0.11 & $\mathrm{M} \times \mathrm{H}$ & $13.61 *$ & 5.33 & 5.2 & $-15.85^{*}$ & $\mathrm{H} \times \mathrm{M}$ & $-11.75^{*}$ & $-15.10^{*}$ & $-19.54 *$ \\
\hline PVB $-2 \times$ PC & $0.85^{*}$ & $\mathrm{M} \times \mathrm{L}$ & $111.72 *$ & 18.75 & 1.2 & $24.66^{*}$ & $\mathrm{H} \times \mathrm{L}$ & $59.59 *$ & $8.14 *$ & 2.48 \\
\hline CLN138A $\times$ CLN138B & $0.80^{*}$ & $\mathrm{~L} \times \mathrm{H}$ & $108.92 *$ & $66.95^{*}$ & $31.20^{*}$ & 0.26 & $\mathrm{~L} \times \mathrm{H}$ & $14.73^{*}$ & -4.58 & -4.58 \\
\hline CLN $138 \mathrm{~A} \times$ CLN 104 & $-0.42 *$ & $\mathrm{~L} \times \mathrm{L}$ & $27.94 *$ & 21.79 & $-36.80 *$ & $8.26^{*}$ & $\mathrm{~L} \times \mathrm{L}$ & $44.19^{*}$ & $37.44 *$ & -8.82 \\
\hline CLN 138A $\times$ CLN 17 & 0.13 & $\mathrm{~L} \times \mathrm{L}$ & $63.90^{*}$ & $41.67^{*}$ & -9.2 & $5.33^{*}$ & $\mathrm{~L} \times \mathrm{M}$ & $18.83^{*}$ & 5.98 & $-10.29 *$ \\
\hline CLN 138A × 2123D & $0.40^{*}$ & $\mathrm{~L} \times \mathrm{L}$ & $91.56^{*}$ & $88.89 *$ & -9.2 & $19.17 *$ & $\mathrm{~L} \times \mathrm{L}$ & $50.63 *$ & $47.29 *$ & -2.28 \\
\hline CLN $138 \mathrm{~A} \times 2123 \mathrm{C}$ & $0.64 *$ & $\mathrm{~L} \times \mathrm{L}$ & $121.50^{*}$ & $102.86^{*}$ & -5.2 & 2.93 & $\mathrm{~L} \times \mathrm{L}$ & $23.44 *$ & $16.75^{*}$ & $-22.55 *$ \\
\hline CLN 138A $\times$ PVB-1 & -0.07 & $\mathrm{~L} \times \mathrm{M}$ & $35.67 *$ & 8.29 & $-14.80 *$ & $-12.52 *$ & $\mathrm{~L} \times \mathrm{H}$ & -1.03 & $-14.89^{*}$ & $-21.57 *$ \\
\hline CLN $138 \mathrm{~A} \times$ CLN 154 & $0.61 *$ & $\mathrm{~L} \times \mathrm{H}$ & $68.94 *$ & $24.00^{*}$ & $24.00^{*}$ & $22.65^{*}$ & $\mathrm{~L} \times \mathrm{M}$ & $41.40^{*}$ & $24.25^{*}$ & $8.82 *$ \\
\hline $\mathrm{CLN} 138 \mathrm{~A} \times \mathrm{PC}$ & $-0.47^{*}$ & $\mathrm{~L} \times \mathrm{L}$ & 35.66 & -17.14 & $-61.20 *$ & $-8.31 *$ & $\mathrm{~L} \times \mathrm{L}$ & $21.57^{*}$ & -8.37 & $-39.22 *$ \\
\hline CLN 138B $\times$ CLN 104 & -0.15 & $\mathrm{H} \times \mathrm{L}$ & $40.67 *$ & 16.95 & -8 & $28.58^{*}$ & $\mathrm{H} \times \mathrm{L}$ & $57.96^{*}$ & $26.47 *$ & $26.47 *$ \\
\hline CLN 138B $\times$ CLN 17 & -0.01 & $\mathrm{H} \times \mathrm{L}$ & $44.54 *$ & $31.19^{*}$ & 3.2 & $-3.52 *$ & $\mathrm{H} \times \mathrm{M}$ & 4.42 & -3.59 & -3.6 \\
\hline CLN 138B $\times 2123 \mathrm{D}$ & $0.65^{*}$ & $\mathrm{H} \times \mathrm{L}$ & $87.38^{*}$ & $50.85^{*}$ & $18.80^{*}$ & 1.83 & $\mathrm{H} \times \mathrm{L}$ & $17.60^{*}$ & -3.92 & -3.92 \\
\hline CLN 138B $\times 2123 \mathrm{C}$ & $1.02 *$ & $\mathrm{H} \times \mathrm{L}$ & $117.69^{*}$ & $62.71 *$ & $28.00^{*}$ & $16.91 *$ & $\mathrm{H} \times \mathrm{L}$ & $33.89 *$ & $6.54 *$ & $6.54 *$ \\
\hline CLN 138B × PVB-1 & $0.41^{*}$ & $\mathrm{H} \times \mathrm{M}$ & $55.84 *$ & $55.67 *$ & $22.80 *$ & $9.13 *$ & $\mathrm{H} \times \mathrm{H}$ & $19.72 *$ & $15.03 *$ & $15.03 *$ \\
\hline CLN 138B $\times$ CLN 154 & -0.14 & $\mathrm{H} \times \mathrm{H}$ & $25.28 *$ & 12 & 12 & $-22.03 *$ & $\mathrm{H} \times \mathrm{M}$ & $-14.28 *$ & $-19.61 *$ & $-19.61 *$ \\
\hline $\mathrm{CLN} 138 \mathrm{~B} \times \mathrm{PC}$ & 0.18 & $\mathrm{H} \times \mathrm{L}$ & $85.65^{*}$ & 5.08 & $-17.20 *$ & $9.34 *$ & $\mathrm{H} \times \mathrm{L}$ & $39.85^{*}$ & $-6.54 *$ & $-6.54 *$ \\
\hline CLN $104 \times$ CLN 17 & $0.36^{*}$ & $\mathrm{~L} \times \mathrm{L}$ & $70.34 *$ & $54.17 *$ & -1.2 & $-14.85^{*}$ & $\mathrm{~L} \times \mathrm{M}$ & 1.14 & $-13.51^{*}$ & $-26.79 *$ \\
\hline CLN $104 \times 2123 \mathrm{D}$ & $0.45^{*}$ & $\mathrm{~L} \times \mathrm{L}$ & $82.40 *$ & $75.64 *$ & -8.8 & $19.83 *$ & $\mathrm{~L} \times \mathrm{L}$ & $64.56^{*}$ & $60.31 *$ & 1.64 \\
\hline CLN $104 \times 2123 \mathrm{C}$ & $0.54^{*}$ & $\mathrm{~L} \times \mathrm{L}$ & $96.48^{*}$ & $71.79 *$ & -10.8 & $16.91 *$ & $\mathrm{~L} \times \mathrm{L}$ & $58.36^{*}$ & $57.07 *$ & -5.56 \\
\hline CLN $104 \times$ PVB-1 & -0.21 & $\mathrm{~L} \times \mathrm{M}$ & $19.88^{*}$ & -0.68 & $-21.60 *$ & $-3.54 *$ & $\mathrm{~L} \times \mathrm{H}$ & $18.88^{*}$ & -1.77 & $-9.48 *$ \\
\hline CLN $104 \times$ CLN 154 & $0.44 *$ & $\mathrm{~L} \times \mathrm{H}$ & $52.63 *$ & $16.00^{*}$ & $16.00^{*}$ & $-9.37 *$ & $\mathrm{~L} \times \mathrm{M}$ & $9.29 *$ & $-7.84 *$ & $-19.28 *$ \\
\hline CLN $104 \times$ PC & $0.40^{*}$ & $\mathrm{~L} \times \mathrm{L}$ & $130.77^{*}$ & $38.46^{*}$ & $-28.00 *$ & $7.67^{*}$ & $\mathrm{~L} \times \mathrm{L}$ & $70.04 *$ & $32.61 *$ & $-20.26^{*}$ \\
\hline CLN $17 \times 2123 \mathrm{D}$ & 0.18 & $\mathrm{~L} \times \mathrm{L}$ & $53.57 *$ & $34.38 *$ & -14 & $-6.27 *$ & $\mathrm{M} \times \mathrm{L}$ & 4.64 & $-8.49 *$ & $-22.55 *$ \\
\hline CLN $17 \times 2123 \mathrm{C}$ & 0.1 & $\mathrm{~L} \times \mathrm{L}$ & $50.19 *$ & 20.83 & $-22.80 *$ & $-4.85 *$ & $\mathrm{M} \times \mathrm{L}$ & 3.64 & $-11.97 *$ & $-25.49 *$ \\
\hline CLN $17 \times$ PVB-1 & $0.71^{*}$ & $\mathrm{~L} \times \mathrm{M}$ & $69.19 *$ & $53.13 *$ & $20.80^{*}$ & $8.03 *$ & $\mathrm{M} \times \mathrm{H}$ & $16.82 *$ & $12.06^{*}$ & 3.26 \\
\hline CLN $17 \times$ CLN 154 & -0.13 & $\mathrm{~L} \times \mathrm{H}$ & $20.49 *$ & -1.33 & -1.2 & $13.87 *$ & $\mathrm{M} \times \mathrm{M}$ & $21.83 *$ & $19.78 *$ & 4.9 \\
\hline CLN $17 \times$ PC & -0.17 & $\mathrm{~L} \times \mathrm{L}$ & $47.31 *$ & -14.58 & $-45.20 *$ & $5.24 *$ & $\mathrm{M} \times \mathrm{L}$ & $33.15^{*}$ & $-6.95 *$ & $-21.25 *$ \\
\hline $2123 \mathrm{D} \times 2123 \mathrm{C}$ & $-0.61 *$ & $\mathrm{~L} \times \mathrm{L}$ & -12.44 & -20.83 & $-62.00 *$ & $-15.50 *$ & $\mathrm{~L} \times \mathrm{L}$ & -4.53 & -7.73 & $-41.50 *$ \\
\hline $2123 \mathrm{D} \times \mathrm{PVB}-1$ & $-0.27 *$ & $\mathrm{~L} \times \mathrm{M}$ & 11.67 & -10.32 & $-29.20 *$ & $-9.29 *$ & $\mathrm{~L} \times \mathrm{H}$ & 3.78 & $-12.41 *$ & $-19.28 *$ \\
\hline $2123 \mathrm{D} \times \mathrm{CLN} 154$ & $0.24 *$ & $\mathrm{~L} \times \mathrm{H}$ & $38.92 *$ & 2.67 & 2.8 & $14.55^{*}$ & $\mathrm{~L} \times \mathrm{M}$ & $32.47 *$ & $14.18^{*}$ & 0 \\
\hline $2123 \mathrm{D} \times \mathrm{PC}$ & -0.14 & $\mathrm{~L} \times \mathrm{L}$ & $53.42 *$ & -6.39 & $-55.20 *$ & 0.59 & $\mathrm{~L} \times \mathrm{L}$ & $41.41^{*}$ & 8.25 & $-31.37 *$ \\
\hline $2123 \mathrm{C} \times \mathrm{PVB}-1$ & $-0.54 *$ & $\mathrm{~L} \times \mathrm{M}$ & -7.48 & $-30.80 *$ & $-45.60 *$ & -3.03 & $\mathrm{~L} \times \mathrm{H}$ & $9.08 *$ & $-10.46^{*}$ & $-17.48 *$ \\
\hline $2123 \mathrm{C} \times \mathrm{CLN} 154$ & $-0.32 *$ & $\mathrm{~L} \times \mathrm{H}$ & 7.78 & $-25.33^{*}$ & $-25.20 *$ & $6.44^{*}$ & $\mathrm{~L} \times \mathrm{M}$ & $19.56^{*}$ & 0.15 & $-12.28 *$ \\
\hline $2123 \mathrm{C} \times \mathrm{PC}$ & $-0.52 *$ & $\mathrm{~L} \times \mathrm{L}$ & -0.81 & $-36.90 *$ & $-75.60 *$ & 1.34 & $\mathrm{~L} \times \mathrm{L}$ & $40.14 *$ & 9.94 & $-34.97 *$ \\
\hline PVB-1 × CLN 154 & 0.01 & $\mathrm{M} \times \mathrm{H}$ & $19.46^{*}$ & 6.67 & 6.8 & 2.85 & $\mathrm{H} \times \mathrm{M}$ & $11.63 *$ & $8.87^{*}$ & 0.32 \\
\hline $\mathrm{PVB}-1 \times \mathrm{PC}$ & $0.70^{*}$ & $\mathrm{M} \times \mathrm{L}$ & $106.28 *$ & 16.75 & -8 & $10.40 *$ & $\mathrm{H} \times \mathrm{L}$ & $43.12 *$ & -2.3 & $-9.97 *$ \\
\hline CLN $154 \times$ PC & $0.31 *$ & $\mathrm{H} \times \mathrm{L}$ & $59.42 *$ & -12 & -12 & -1.15 & $\mathrm{M} \times \mathrm{L}$ & $23.11 *$ & $-14.79 *$ & $-25.37 *$ \\
\hline$* * \mathrm{CD}(P \leq 0.05)$ & 0.21 & - & 0.31 & 0.36 & 0.36 & 3.34 & - & 4.96 & 5.73 & 5.73 \\
\hline $\mathrm{SE}\left(\mathrm{s}_{\mathrm{ij}}\right)$ & 0.12 & & & & & 1.9 & & & & \\
\hline $\mathrm{SE}\left(\mathrm{s}_{\mathrm{ij}}-\mathrm{s}_{\mathrm{ik}}\right)$ & 0.17 & & & & & 2.8 & & & & \\
\hline $\mathrm{SE}\left(\mathrm{s}_{\mathrm{ij}}-\mathrm{S}_{\mathrm{kl}}\right)$ & 0.16 & & & & & 2.67 & & & & \\
\hline
\end{tabular}

*Significant at $P \leq 0.05,{ }^{*}$ Critical difference (CD) 


\section{Discussion}

The cultivated tomato has provided little resistance to the ToLCV (Pico et al., 1998; Ji et al., 2007a). Tolerance was found in Nova (Mayee et al., 1974) and EC 104395 (Varma et al., 1980). Breeding begomovirus-resistant cultivars have involved the introgression of resistance genes from wild tomato species. Among the wild species $L$. hirsutum $f$ glabratum and L. peruvianum were highly resistant (Banerjee and Kalloo, 1987). Resistance in L. pimpinellifolium LA1921 was found to be monogenic and incompletely dominant, and two epistatic genes governed resistance in L. hirsutum $f$ glabratum (B 6013). Two independent genes for resistance seem to be involved in these two-wild species with that of L. hirsutum $f$ glabaratum dominant over the other (Banerjee and Kalloo, 1990). Pilowsky and Cohen (1974) studied crosses between a tomato yellow leaf curl virus (ToYLCV) homozygous resistant line of $L$. pimpinellifolium and the susceptible tomato cv Pearson. Inheritance of ToYLCV was studied by Hassan et al. (1984) in the interspecific crosses between L. esculentum Mill. cv UC $82 \times$ L. cheesmanii ssp. minor (Hook) C H Mill. cv LA 1401, and L. esculentum cv. VF 145-B-7879 $\times$ L. hirsutum Humb. \& Bonpl. cv LA 386. They artificially inoculated the genetic population with ToYLCV before transplanting and later evaluated under field conditions. They found that resistance derived from $L$. cheesmanii seems to be recessive, and resistance derived from L. hirsutum is dominant and controlled by more than one gene. Dhaliwal and Cheema (2011) have also reported 20.93-58.75\% heterosis over better parent for total yield in promising F1 hybrids tested under ToLCV infested conditions. The high yield and disease resistance of F1 hybrids must be accompanied by good horticultural traits like shape, size, flavour etc. for them to become commercially acceptable. Furthermore, these results are in accordance with the result for the other member of Solanaceae, i.e. eggplant (Kaushik et al., 2018; Kaushik 2019)

Banerjee and Kalloo (1987) studied the inheritance of ToLCV resistance in interspecific crosses between ToLCV resistant L. hirsutum f glabratum line B6013 and five susceptible cultivars (HS101, HS102, HS110, Pusa Ruby and Punjab Chhuhara) of L. esculentum. They reported that resistance derived from L. hirsutm f glabratum B6013 based on two epistatic genes one from the wild parent and another from the cultivated one. The inheritance of tolerance of ToYLCV, deriving from the wild tomato $L$. peruvianum, was studied by Pilowsky and Cohen (1990) crossing the cultivated tomato (L. esculentum Mill.) line M-60 (ToYLCV-tolerant) with line M-10 (ToYLCVsusceptible line). Genetic data indicated that 5 recessive genes controlled tolerance. Nagaraja et al. (1996) estimated the gene effects for fruit yield, its components and inheritance of leaf curl virus resistance in two interspecific crosses between $L$. esculentum cultivars Arka Saurabh and Arka Vikas and tomato leaf curl virus resistant L. hirsutum. They concluded that an additive-dominance model accounted for most of the variation among generations for days to tomato leaf curl virus symptoms expression in both the crosses.

The inheritance of resistance to ToLCV disease was assessed by Nainar and Pappiah (2002) under field conditions in eight crosses involving susceptible tomato cultivars PKM1, CO.2, CO.3 and Pusa Ruby and resistant wild parent's L. hirsutum and $L$. pimpinellifolium. They reported that the resistance in L. hirsutum was controlled by three recessive genes and a single incomplete dominant gene in L. pimpinellifolium.

Shekara et al. (2003) studied the inheritance of resistance to tomato leaf curl virus in tomato using triple test cross analysis. The genotypes consisted of crosses between 148 
resistant testers and the susceptible cultivar Arka Abha and crosses between the resistant cultivar ATY-1 and the susceptible cultivars Arka Sourabh. The importance of the additive genetic variance for resistance was observed. The magnitude of the additive genetic component was higher than the dominance component and suggested pedigree selection method for the development of cultivars resistant to tomato leaf curl virus.

Resistance to ToYLCV was studied by Castro et al. (2007) in a cross between $S$. lycopersicum $\times S$. pimpinellifolium UPV16991. Crosses between four breeding lines susceptible to ToYLCV and L102 were also performed to study the dominance of the resistance in $S$. lycopersicum genetic backgrounds. Response to ToYLCV infection of $\mathrm{P}_{1}, \mathrm{P}_{2}, \mathrm{~F}_{1}, \mathrm{~F}_{2}, \mathrm{BC}_{1}$, and $\mathrm{BC}_{2}$ generations fitted, for this line, a monogenic control with partial recessiveness and incomplete penetrance. Hazra and Nath (2008) conducted an experiment to evaluate 25 tomato genotypes for resistance to tomato leaf curl virus and concluded that percent disease incidence and co-efficient of infection in the genotypes was highest in early autumn (planting in last week of August), followed by springsummer (planting in first week of February) and autumn-winter (planting in second week of October) seasons. The extent of heterosis for tospovirus resistance, yield and associated characters was studied by Kumar et al. (2009) by using line $\times$ tester mating. The lines carrying Ty-2 gene have been reported to be susceptible to bipartite tomatoinfecting begomoviruses (Giordano et al., 1998; Prasanna et al., 2015).

Ji and Scott (2006) mapped Ty-3 gene on the long arm of chromosome 6, between cLED-31-P16 and T1079 markers. Recently, Kadirvel et al. (2013) identified new locus (qTy10.1) on chromosome 10 in the line FLA456, named it as Ty-6. Similar to our results a series of lines pyramided by Prasanna et al. (2014) using $S$. habrochaites derived resistance $(T y-2)$, and $S$. chilense derived resistance $(T y-3)$. The pyramided lines and $T y-3$ carrying lines exhibited a high level of resistance that could be used as critical genetic stocks for resistance breeding. Also, Shankarappa et al. (2008) developed two hybrids viz., BLRH-3 and BLRH-16 resistant to ToLCV both in the glasshouse and the field conditions. Based on phenotypic survey and virus quantification two highly resistant hybrids SJ12 and RFT112 were identified (Tabein et al., 2017). Recently, Elbaz et al. (2016) found that the entries with Ty-1/Ty-3 + Ty-2 offered the highest levels of resistance to TYLCV. Whereas, Rubio et al. (2016) determined and quantified the effect of Ty-1 gene introgression on yield and quality of tomatoes. Ty-1 introgression negatively affected the yield attributes.

Tomás et al. (2011) screened for ToYLCVD resistance under high disease pressure generated by natural infection in the field. They have concluded two independent loci, one dominant and one recessive, were associated with EELM- 889 resistance. The study showed these loci to be distinct from that of the resistance gene $(T y-1)$ deployed in resistant tomato cultivars. Therefore, both kinds of resistance could be combined to provide improved resistance to ToYLCVD. Dhaliwal and Cheema (2011) have also reported that hybrids were manifesting significant standard heterosis for yield in tomato also expressed significant standard heterosis for fruit weight. Therefore, it is advisable to handle these cross-combinations by pedigree selection to exploit additive gene action to find significant desirable improvement in yield along with resistance to ToLCV (Dhaliwal and Cheema, 2011). In our study, the hybrids identified should not be resorted to selection in segregating generations as their superior performance is attributed to the involvement of non-fixable gene effects. 


\section{Conclusions}

Extensive and significant difference in the performance of lines carrying different combinations of resistance genes (Ty-X) against ToLCV. The top parent CLN 154 yielding significantly higher than released varieties along with seven $F_{1}$ hybrids manifesting 16 to $31 \%$ top parent heterosis for total yield can be recommended after multi-location evaluations. Overall, this study provides useful information on the successful leaf curl virus resistant gene combinations under the north-Indian conditions.

Acknowledgements. Authors are thankful to AVRDC - The World Vegetable Centre, Taiwan, for kindly providing the seeds of CLN lines having variable degree of resistance to ToLCV.

\section{REFERENCES}

[1] Banerjee, M. K., Kalloo, G. (1987a): Inheritance of resistance to tomato leaf curl virus in Lycopersicon hirsutum f. glabratum. - Euphytica 36: 581-84.

[2] Banerjee, M. K., Kalloo, G. (1987b): Sources and inheritance of resistance to leaf curl virus in Lycopersicon. - Theoretical and Applied Genetics 73: 707-10.

[3] Banerjee, M. K., Kalloo, G. (1990): Nature of resistance to tomato leaf curl virus (TLCV) in two species of Lycopersicon. - Haryana Agricultural University Journal of Research 20: 225-28.

[4] Castro, P. A., Diez, M. J., Nuez, F. (2007): Inheritance of tomato yellow leaf curl virus resistance derived from Solanum pimpinellifolium UPV16991. - Plant Disease 91: 87985 .

[5] Cheema, D. S., Dhaliwal, M. S. (2010): Punjab Varkha Bahar 1 and Punjab Varkha Bahar 2: New varieties of tomato resistant to ToYLCV. - Journal of Research, Punjab Agricultural University 47: 116-117.

[6] Elbaz, M., Hanson, P., Fgaier, S., Laarif, A. (2016): Evaluation of tomato entries with different combinations of resistance genes to tomato yellow leaf curl disease in Tunisia. Plant Breed. 55(6): 1-7.

[7] FAOSTAT (2018): http://www.fao.org/faostat/en/\#data/QC. - Accessed 12.11.18.

[8] Gaikwad, K. A., Cheema, D. S., Sharma, A., Dhaliwal, M. S. (2011a): Reaction of elite tomato (Solanum lycopersicum L.) germplasm against tomato leaf curl virus disease. Acta Horticulturae 157-161. https://doi.org/10.17660/ActaHortic.2011.914.28.

[9] Gaikwad, K. A., Sharma, A., Cheema, D. S. (2011b): Molecular detection and characterization of leaf curl virus infecting tomato in Punjab India. - Acta Horticulturae 153-156. https://doi.org/10.17660/ActaHortic.2011.914.27.

[10] Garg, N., Cheema, D. S. (2011): Assessment of fruit quality attributes of tomato hybrids involving ripening mutants under high temperature conditions. - Scientia Horticulturae 131: 29-38.

[11] Giordano, L. de B., Bezerra, I. C., Ferreira, P. T. O., Borges Neto, C. R. (1998): Breeding tomatoes for resistance to whitefly-transmitted geminivirus with bipartite genome in Brazil. - VI International Symposium on Processing Tomato \& Workshop on Irrigation \& Fertigation of Processing Tomato, pp. 357-360.

[12] Griffing, B. (1956): Concept of general and specific combining ability in relation to diallel crossing systems. Australian Journal of Biological Sciences - 9: 463-493. https://doi.org/10.1071/bi9560463.

[13] Hanson, P., Green, S. K., Kuo, G. (2006): Ty-2, a gene on chromosome 11 conditioning geminivirus resistance in tomato. - Tomato Genetics Cooperative Report 56: 17-18.

[14] Hanson, P., Lu, S.-F., Wang, J.-F., Chen, W., Kenyon, L., Tan, C.-W., Tee, K. L., Wang, Y. Y., Hsu, Y. C., Schafleitner, R. (2016): Conventional and molecular marker-assisted 
selection and pyramiding of genes for multiple disease resistance in tomato. - Scientia Horticulturae 201: 346-354.

[15] Hassan, A. A., Laterrot, H., Mazyed, H. M., Nassor, S. H., Sims, W. L., Nakhla, M. K. (1984): Genetics and heritability of tomato yellow leaf curl virus tolerance derived from Lycopersicon pimpinellifolium. - European Assn. for Res. on Plant Breeding. Tomato Working Group, Wageningen, Netherlands.

[16] Hazra, P., Nath, S. (2008): Source of resistance in tomato (Lycopersicon esculentum Mill.) and inheritance of host resistance for tomato leaf curl disease. - Indian Journal of Agricultural Sciences 78: 690-94.

[17] Hong, Y. G., Harrison, B. D. (1995): Nucleotide sequences from tomato leaf curl viruses from different countries: evidence for three geographically separate branches in evolution of the coat protein of whitefly-transmitted geminiviruses. - Journal of General Virology 76(Pt 8): 2043-2049. https://doi.org/10.1099/0022-1317-76-8-2043.

[18] Horowitz, A. R., Kontsedalov, S., Khasdan, V., Ishaaya, I. (2005): Biotypes B and Q of Bemisia tabaci and their relevance to neonicotinoid and pyriproxyfen resistance. Archives of Insect Biochemistry and Physiology 58: 216-225. https://doi.org/10.1002/arch.20044.

[19] Hutton, S. F., Scott, J. W. (2014): Ty-6, a major begomovirus resistance gene located on chromosome 10. - Tomato Genetics Cooperative Report 64: 14-18.

[20] Hutton, S. F., Scott, J. W., Schuster, D. J. (2012): Recessive resistance to tomato yellow leaf curl virus from the tomato cultivar Tyking is located in the same region as Ty-5 on Chromosome 4. - HortScience 47: 324-327.

[21] Ji, Y. F., Scott, J. W., Hanson, P., Graham, E., Maxwell, D. P. (2007a): Sources of Resistance, Inheritance and Location of Genetic Loci Conferring Resistance to Members of the Tomato-Infecting Begomoviruses. - In: Czosnek, H. (ed.) Tomato Yellow Leaf Curl Disease, Springer, Dordrechte, pp: 343-62.

[22] Ji, Y., Schuster, D. J., Scott, J. W. (2007b): Ty-3, a begomovirus resistance locus near the Tomato yellow leaf curl virus resistance locus Ty-1 on chromosome 6 of tomato. Molecular Breeding 20: 271-284.

[23] Ji, Y., Scott, J. W., Schuster, D. J., Maxwell, D. P. (2009): Molecular mapping of Ty-4, a new tomato yellow leaf curl virus resistance locus on chromosome 3 of tomato. - Journal of the American Society for Horticultural Science 134: 281-288.

[24] Kadirvel, P., de la Pana, R., Schaffeitner, R., Huang, S., Geethanjali, S., Kenyon, L., Tsai, W., and Hanson, P. (2013): Mapping of QTLs in tomato line FLA456 associated with resistance to a virus causing yellow leaf curl disease. - Euphytica 190: 297-308.

[25] Kaushik, P., Dhaliwal, M. S. (2018): Diallel analysis for morphological and biochemical traits in tomato cultivated under the influence of tomato leaf curl virus. - Agronomy 8: 153. https://doi.org/10.3390/agronomy8080153.

[26] Kaushik, P., Dhaliwal, S. M., Jindal, K. S., Srivastava, A., Tyagi, V., Brar, S. N., Rana, K. M. (2015): Heterosis and leaf curl virus resistance in rainy season tomato under North Indian conditions. - African Journal of Agricultural Research 10: 2763-2772. https://doi.org/10.5897/AJAR2014.9133.

[27] Kaushik, P., Plazas, M., Prohens, J., Vilanova, S., Gramazio, P. (2018): Diallel genetic analysis for multiple traits in eggplant and assessment of genetic distances for predicting hybrids performance. $\quad-\quad$ Plos One 13: e0199943. https://doi.org/10.1371/journal.pone.0199943.

[28] Kaushik, P. (2019): Line $\times$ Tester Analysis for Morphological and Fruit Biochemical Traits in Eggplant (Solanum melongena L.) Using Wild Relatives as Testers. Agronomy 9: 185. doi:10.3390/agronomy9040185.

[29] Kirthi, N., Maiya, S. P., Murthy, M. R. N., Savithri, H. S. (2002): Evidence for recombination among the tomato leaf curl virus strains/species from Bangalore, India. Archives of Virology 147: 255-272. 
[30] Kumar, K. H. Y., Patil, S. S., Dharmatti, P. R., Byadagi, A. S., Kajjidoni, S. T., Patil, R. H. (2009): Estimation of heterosis for tospovirus resistance in tomato. - Karnataka Journal of Agricultural Sciences 22: 1073-75.

[31] Mayee, C. D., Kanwar, J. S., Nandpuri, K. S. (1974): The comparative performance of different genotypes of tomato vis-a-vis leaf curl and mosaic. - Journal of Research (PAU) 11: 362-64.

[32] Moriones, E., Praveen, S., Chakraborty, S. (2017): Tomato Leaf Curl New Delhi Virus: An emerging virus complex threatening vegetable and fiber crops. - Viruses 9. https://doi.org/10.3390/v9100264.

[33] Muniyappa, V., Venkatesh, H. M., Ramappa, H. K., Kulkarni, R. S., Zeidan, M., Tarba, C. Y., Ghanim, M., Czosnek, H. (2000): Tomato leaf curl virus from Bangalore (ToLCVBan4): sequence comparison with Indian ToLCV isolates, detection in plants and insects, and vector relationships. - Archives of Virology 145: 1583-1598.

[34] Nagaraja, T. E., Kulkarni, R. S., Prasad, S. G., Basavaraja, G. T. (1996): Estimates of gene effects for fruit yield its components and inheritance in two interspecific crosses of tomato. - Crop Improvement 23: 71-74.

[35] Nainar, P., Pappiah, C. M. (2002): Inheritance of resistance to tomato leaf curl virus disease (ToLCV) in the segregating generations of eight crosses of tomato (Lycopersicon esculentum Mill.). - South Indian Horticulture 50: 72-77.

[36] Pico, B. M., Diez, J., Nuez, F. (1998): Evaluation of whitefly-mediated inoculation techniques to screen Lycopersicon esculenlum and wild relatives for resistance to tomato yellow leaf curl virus. - Euphytica 101: 259-71.

[37] Picó, B., Díez, M. J., Nuez, F. (1996): Viral diseases causing the greatest economic losses to the tomato crop. II. The tomato yellow leaf curl virus - a review. - Scientia Horticulturae 67: 151-196. https://doi.org/10.1016/S0304-4238(96)00945-4.

[38] Pilowsky, M., Cohen, S. (1974): Inheritance of resistance to tomato yellow leaf curl virus in tomatoes. - Phytopathology 64: 632-672.

[39] Pilowsky, M., Cohen, S. (1990): Tolerance to tomato yellow leaf curl virus derived from Lycopersicon peruvianum. - Plant Disease 4: 248-50.

[40] Prasanna, H. C., Sinha, D. P., Rai, G. K., Krishna, R., Kashyap, S. P., Singh, N. K., Singh, M., Malathi, V. G. (2014): Pyramiding Ty-2 and Ty-3 genes for resistance to monopartite and bipartite tomato leaf curl viruses of India. - Plant Pathol. 10: 1-10.

[41] Prasanna, H. C., Kashyap, S. P., Krishna, R., Sinha, D. P., Reddy, S., Malathi, V. G. (2015): Marker assisted selection of Ty-2 and Ty-3 carrying tomato lines and their implications in breeding tomato leaf curl disease resistant hybrids. - Euphytica 204: 407418. https://doi.org/10.1007/s10681-015-1357-8.

[42] Rubio, F., Alonso, A., Martínez, S. G., Ruiz, J. R. (2016): Introgression of virusresistance genes into traditional Spanish tomato cultivars (Solanum lycopersicum L.): Effects on yield and quality. - Scientia Horticulturae 198: 183-190.

[43] Shankarappa, K. S., Sriharsha, Rangaswamy, K. T., Aswathanarayana, D. S., Prameela, H. A., Kulkarni, R. S., Muniyappa, V., Rao, A. M., Maruthi, M. N. (2008): Development of tomato hybrids resistant to tomato leaf curl virus disease in South India. - Euphytica 164: 531-539. https://doi.org/10.1007/s10681-008-9750-1.

[44] Sharma, J. R. (2006): Statistical and Biometrical Techniques in Plant Breeding. - New Age International Publisher, New Delhi.

[45] Shekara, A. C. C., Kulkarni, R. S., Muniyappa, V. (2003): Triple test cross analysis of tomato leaf curl virus resistance in tomato (Lycopersicon esculentum Mill.). - Indian Journal of Genetics 63: 185-86.

[46] Singh, R. K., Rai, N., Singh, M. J. I., Singh, S. N., Srivastava, K. (2014): Genetic analysis to identify good combiners for ToLCV resistance and yield components in tomato using interspecific hybridization. - J Genet 93(3): 623-629. 
[47] Singh, R. K., Rai, N., Singh, M., Saha, S., Singh, S. N. (2015): Detection of tomato leaf curl virus resistance and inheritance in tomato (Solanum lycopersicum L.). - The Journal of Agricultural Science 153: 78-89. https://doi.org/10.1017/S0021859613000932.

[48] Tabein, S., Behjatnia, S. A., Laviano, L., Pecchioni, N., Accotto, G. P., Noris, E., Miozzi, L. (2017): Pyramiding Ty-1/Ty-3 and Ty-2 in tomato hybrids dramatically inhibits symptom expression and accumulation of tomato yellow leaf curl disease inducing viruses. - Arch. Phytopathol. Plant Prot. 315: 1-15.

[49] Tomás, D. M., Cañizares, M. C., Abad, J., Muñoz, R. F., Moriones, E. (2011): Resistance to Tomato yellow leaf curl virus accumulation in the tomato wild relative Solanum habrochaites associated with the $\mathrm{C} 4$ viral protein. - Molecular Plant-Microbe Interactions Journal 24: 849-61.

[50] Varma, A., Malathi, V. G. (2003): Emerging geminivirus problems: a serious threat to crop production. - Annals of Applied Biology 142: 145-164.

[51] Verlaan, M. G., Hutton, S. F., Ibrahem, R. M., Kormelink, R., Visser, R. G. F., Scott, J. W., Edwards, J. D., Bai, Y. (2013): The Tomato Yellow Leaf Curl Virus resistance genes Ty-1 and Ty-3 are allelic and code for DFDGD-Class RNA-dependent RNA $\begin{array}{llllll}\text { Polymerases. } & - & \text { PLOS } & \text { Genetics } & \text { 9: }\end{array}$ https://doi.org/10.1371/journal.pgen.1003399.

[52] Zamir, D., Ekstein-Michelson, I., Zakay, Y., Navot, N., Zeidan, M., Sarfatti, M., Eshed, Y., Harel, E., Pleban, T., van-Oss, H., Kedar, N., Rabinowitch, H. D., Czosnek, H. (1994): Mapping and introgression of a tomato yellow leaf curl virus tolerance gene, TY1. - Theoretical and Applied Genetics 88: 141-146. https://doi.org/10.1007/BF00225889. 